\section{Cahiers de Narratologie}

Analyse et théorie narratives

10.2 | 2001

La voix narrative

\title{
Voix de l'auteur/voix du lecteur : analyse sémiotique d'un poème de Yoshioka Minoru
}

\section{J. A. F. Hopkins}

\section{(2) OpenEdition}

1 Journals

Édition électronique

URL : http://journals.openedition.org/narratologie/10180

DOI : 10.4000/narratologie.10180

ISSN : 1765-307X

Éditeur

LIRCES

\section{Édition imprimée}

Date de publication : 1 janvier 2001

Pagination : 69-74

ISBN : 2914561032

ISSN : $0993-8516$

\section{Référence électronique}

J. A. F. Hopkins, « Voix de l'auteur/voix du lecteur : analyse sémiotique d'un poème de Yoshioka Minoru », Cahiers de Narratologie [En ligne], 10.2 | 2001, mis en ligne le 01 janvier 2001, consulté le 11 juin 2020. URL : http://journals.openedition.org/narratologie/10180 ; DOI : https://doi.org/10.4000/ narratologie.10180 


\title{
VOIX DE L'AUTEUR/VOIX DU LECTEUR : ANALYSE SÉMIOTIQUE D'UN POÈME DE YOSHIOKA MINORU
}

\author{
J. A. F. HOPKINS \\ Université Tamagawa, Tôkyô
}

\section{Introduction}

Je voudrais parler de la voix de l'auteur et de celle du lecteur dans la poésie moderne - celle depuis Baudelaire. Mes remarques ne se limiteront point à la poésie française : en effet mon exemple principal est puisé dans la littérature japonaise.

A la différence de la littérature narrative, ou de la poésie romantique ou post-moderne - où l'auteur souvent raconte une histoire à travers les actions de ses caractères - dans la poésie moderne l'auteur a recours à des ensembles d'images pour exprimer indirectement des concepts ou propositions sous-jacents qui n'apparaissent pas sur la surface du texte. Afin d'écouter cette "voix de l'auteur », le lecteur est obligé de faire un travail considérable de comparaison des images d'un ensemble - travail visant la reconstitution de la proposition sous-jacente qui les génère toutes - sans se laisser détourner par les références disparates faites par l'image individuelle au monde extra-textuel.

2. Selon la théorie sémiotique de Michael Riffaterre, sur laquelle je baserai la mienne, les images générées par une même proposition - ou matrice - ne sont pas nécessairement contiguës dans le texte. "L'agrammaticalité » de leur structure superficielle signale qu'elles représentent la matrice (Voir Riffaterre 1983, p. 12)1. La structure sémantique de la matrice a pour autorité une structure intertextuelle analogue,

1 Riffaterre 1983, Sémiotique de la poésie, trad. J.-J. Thomas, Paris: Seuil; basé sur Riffaterre 1978, Semiotics of Poetry, Bloomington : Indiana University Press. 
que le lecteur est obligé de chercher dans d'autres œuvres d'art de sa connaissance.

Cette théorie simplifie trop la structure interne du texte. Dans beaucoup de cas nous avons évidemment affaire à deux matrices, au lieu d'une seule. Les propositions matricielles (« $\mathrm{PM} » \mathrm{~s})$ sont liées entre elles par une relation d'ordre syntagmatique, qui peut prendre plusieurs formes. Il s'ensuit que l'intertexte sera également de structure bimatricielle ${ }^{2}$.

Selon la version riffaterrienne de la triade peircienne de la signification, l'interprétant du texte (en tant que signesujet) et de l'intertexte (signe-objet) serait une deuxième structure intertextuelle capable de «médiatiser la référence du texte à son intertexte »(Riffaterre 1980c, p. 9) ${ }^{3}$.

3. Tandis que c'est la voix de l'auteur qui se laisse apercevoir dans la structure bimatricielle du texte, lorsqu'il en vient au choix d'un intertexte le lecteur a un rôle important à jouer. Bien qu'il faut que l'intertexte soit analogue au texte primaire au niveau de la structure sémiotique, une certaine liberté est laissée au lecteur au niveau lexical. Il s'ensuit que l'intertexte peut varier selon le choix fait par le lecteur de parmi les œuvres d'art disponibles à sa mémoire.

4. La lecture d'un texte moderne met en jeu d'autres mécanismes exigeant encore des contributions de la part du lecteur. Je pense à la capacité de l'œuvre d'art moderne de transformer les préconceptions du lecteur - capacité attestée d'ailleurs par Lotman, Eco, Jameson et F. Ponge ${ }^{4}$. Cette transformation s'opère grâce à une comparaison entre, d'un côté, les propositions novatrices de l'interprétant et de l'intertexte

2 Pour un exposé plus détaillé de la théorie de Riffaterre, voir Hopkins 1994: Présentation et critique de la théorie sémiotique littéraire de Michael Riffaterre, Université Sophia, Tôkyô : Sophia Linguistica 36 (393 à xix p.). 18.

3 Riffaterre 1980c "La trace de l'intertexte », La Pensée 215, p. 4-

4 Voir, par exemple, Eco, U. 1976, A Theory of Semiotics, Bloomington : Indiana University Press, p. 275 ; et Eco 1979/1984, The Role of the Reader, Bloomington: Indiana University Press, p. 194. 
et, de l'autre, les propositions familières d'une contrepartie sociolectique qu'il est du devoir du lecteur de retrouver. La forme lexicale de cette entité est encodée dans l'interprétant, bien que son vocabulaire puisse être plus généralisé. Je l'appellerai le «contexte sociolectique » (CS) du texte, puisqu'il s'agit de l'environnement sociolectique dans lequel le texte présente ses idées novatrices.

Tout en comprenant un vocabulaire semblable à celui du texte et de son interprétant, le CS n'offre qu'une proposition banale par rapport à celle de l'interprétant. Ce dernier réagit immédiatement contre le CS, produisant une transformation des idées reçues du lecteur. C'est sans doute ce dialogue entre texte et lecteur qui, tout en engageant la "voix» de celui-ci, transforme ses habitudes de penser - ce changement constituant l'interprétant final dans la terminologie de Peirce comme elle est expliquée par Eco dans sa The Role of the Reader (1984 : 194 ; voir la note 4).

5. Je voudrais illustrer le fonctionnement de ce mécanisme de transformation à l'aide d'un texte moderne japonais, ce qui démontrera que cette théorie de la lecture ne se limite pas à la littérature occidentale. Ce texte est le court poème Shima (Ile), de Yoshioka Minoru, publié en 19585.

1 Débarquant sur l'île l'homme découvre aux

2 coins des rochers certains grands fragments courbés

3 d'os d'animaux et d'arêtes de poissons le soleil tournant

4 a transformé [blanchi] la tête d'une pieuvre noire en une carte à échelle réduite

5 Aux coins des yeux de l'homme qui retournent à l'horizontal

6 une lune se lève à un angle aigu Essaie d'oublier !

7 Maintenant avec une lucidité extrême l'œuf d'un oiseau de mer

8 s'approche

5 Yoshioka, Minoru 1978, Shin-sen Yoshioka Minoru Shi-shû (Shin-sen Gendai-shi Bunko no.110), Tôkyô : Shichô-sha. Ce poème est également inclus dans le Gendai Nihon Bungaku Taikei, vol. 93, p. 275-276.

La traduction est de mon cru. 
9 Pourquoi n'y a-t-il pas de musique à des moments comme celui-là ?

10 C'est le moment de donner forme à des arcs insomniaques

11 Les mains et les pieds distants de l'homme étendu par terre

12 bougent un peu avec chaque mouvement

13 - de son bord inférieur - la superficie de l'île commence à se contracter

14 Cet endroit est sans doute le nid du soleil du lendemain

15 Pour les oiseaux de l'imagination qui ne voleront jamais

16 l'œuf s'agrandit à côté de l'homme

17 Quel que soit l'endroit où l'on cherche sur sa surface exposée à la lumière violente

18 on ne trouve la moindre marque faite par les ongles

19 des gens aventureux

20 Il ne choisit pas cet Atlas De l'intérieur d'une matrice amaigrie

21 cet homme extrait en tordant un peu de voix et un peu de sang

22 De l'autre côté de cette surface isolatrice

23 les vagues hivernales continuent à glisser

Ayant comparé les deux premières images, l'on s'aperçoit que le composant sémique commun est l'idée de l'objet banal ou familier : contre les efforts de l'imagination que l'homme produit dans les images ultérieures, ces objets trouvés sur l'île peuvent représenter le «matériel » sociolectique qui définit son monde pour l'homme vivant à une étape où il n'a pas encore su définir sa propre existence.

L'apparition agrammaticale (avec une lucidité extrême) d'un $x u f$ d'oiseau de mer - objet confondu avec la lune peu ordinaire du vers précédent - semble refléter l'influence d'une deuxième matrice. Cet «œuf » constitue une forme brillante dans l'imagination de l'homme : une figure pour un idéal inaccessible qu'il peut néanmoins attirer auprès de lui.

La contraction de l'île, en réponse aux mouvements des mains et des pieds de l'homme, exprime assez clairement le concept matriciel que l'homme peut ainsi effectuer des changements dans son environnement. Cette contraction 
permet à l'île de coïncider en superficie avec « l'œuf » qui, lui, s'agrandit.

Dans l'image suivante, si les oiseaux représentent l'humanité et son imagination, c'est alors pour eux, à cause d'eux, que ce phénomène se produit. Si l'île est son nid, l'homme, en tant que locataire, devient une espèce d'oiseau. Le 14ème vers - Cet endroit est le nid du soleil du lendemain - suggère que l'île est justement le point de départ pour des idées nouvelles.

L'exhortation énigmatique du vers 6 - Essaie d'oublier! - peut être admise comme la clef du lien entre nos deux matrices : l'oubli des limitations de son environnement psychologique original permet à l'homme de créer quelque chose avec son imagination même si ce n'est que la «petite voix »du vers 21. Cette "clef » nous aide à construire la structure matricielle de notre texte qui a la forme suivante : C'est précisément en étant la cause de la contraction de son ile - en la changeant en une "lune de l'imagination» (PM2) - que notre homme peut s'isoler du sociolecte et de ses impositions culturelles (PM1), idée que représente cette mer dont les vagues continuent à glisser, au dernier vers. Cette structure bimatricielle constitue l'essentiel de la voix de l'auteur dans ce texte.

\section{Intertexte et interprétant}

Nous passons maintenant au côté de la voix du lecteur, avec ses hypothèses concernant l'intertext et l'interprétant.

En ce qui concerne l'intertexte, il existe des parallèles fascinants entre le poème et les thèmes-clés du film de Stanley Kubrick, 2001, l'Odyssée de l'espace (1968).

Dans le film, on trouve l'analogue de la première matrice de notre poème (à propos des impositions du sociolecte) : le gouvernement terrestre des astronautes leur assigne la tâche de voyager à la lune, afin de rechercher leurs collègues perdus lors d'une expédition antérieure. Là, un monolithe mystérieux les confronte, transmettant des ondes magnétiques vers la planète Jupiter pour laquelle ils se décident à s'embarquer.

Pour résumer la structure bimatricielle de notre intertexte, disons que, dans la matrice du film analogue à notre PM1, nous rencontrons les limites de la technologie terrestre, 
impuissantes devant les mystères de l'espace. Dans l'analogue de la rencontre de notre protagoniste avec l'œuf/lune (PM2), le seul astronaute qui reste vivant s'engage dans une quête qui le mènera loin au-delà des limites de la technologie humaine. Bien que cette dernière paraisse impressionnante en comparaison avec les "petits mouvements » de l'homme du poème, cette technologie - sous la forme d'un ordinateur révolté " HAL »- en effet tue la plupart des astronautes avant que le dernier ne puisse l'arrêter. Il est certain que ce sont ses efforts de résolution et d'imagination qui permettent à l'astronaute d'achever son voyage : ces efforts constituent donc l'essentiel de la relation intermatricielle de l'intertexte.

L'interprétant qui en résulte peut se résumer ainsi : Les limitations que la civilisation sociolectique impose à nos protagonistes (PM1) peuvent être surmontées grâce à l'effort imaginatif exigé de l'individu qui accepte la direction / l'aide d'un agent extra-terrestre (PM2). L'élément novateur ici se trouve dans l'idée essentielle de cette relation intermatricielle : celle qui veut que l'effort imaginatif du protagoniste lui permette de surmonter, de répudier les limitations du sociolecte et d'effectuer par la suite un changement radical dans son environnement.

\section{Contexte sociolectique (ou « CS»)}

Dans le cas de 2001 , le CS est indiqué par le mot Odyssée du titre. L'histoire d'Homère manque l'élément de répudiation du sociolecte qui constitue la condition préalable de la structure matricielle du film et du poème. Ulysse part à la guerre afin de lutter pour son propre monde sociolectique, qu'il ne nie jamais - même s'il semble l'oublier quelquefois au cours de son retour. De plus, il existe une différence importante d'échelle : Ulysse lui-même est apparenté aux dieux, ce fait donnant à l'épopée son aspect héroïque. La structure interne de l'épopée diffère donc de celle de notre interprétant : dans le poème comme dans le film, les efforts du protagoniste sont pénibles; en même temps il faut qu'il répudie son propre sociolecte pour les faire. Le contraste avec le h suggère au lecteur que l'homme appartenant à l'échelon ordinaire et qui veut risquer l'aventure doit accepter la condition de renier son monde familier. 